\title{
Enhancing Immunity and Nutrition: A Vital Topic in Novel COVID-19 Era under Quarantine
}

\author{
Rokayya Sami ${ }^{1}$, Fuguo $\mathrm{Jia}^{1}$, Yang $\mathrm{Li}^{1}$, and Manal Mused Almatrafi ${ }^{2}$ \\ ${ }^{1}$ Northeast Agricultural University \\ ${ }^{2}$ Taif University College of Science
}

May 20, 2020

\begin{abstract}
Coronavirus (COVID-19), as a respiratory disease, has become a serious globally issue (December 2019), China. There is a strong immunenutrition relationship with the respiratory viruses as coronavirus. Moreover, a decline in the immunity function is regularly inevitable with poor micronutrient, inflammation and the declining ( $\mathrm{T}$ cell) function. Nutrition weapon can be one of the main factors for immune response activation. Therefore, there is a globally necessary need to join hands for human survival. This report focuses on the recommendations for maintaining an efficient immune system during quarantine are; getting balanced healthy food, supplements and planning meal times accompanied by light sports for immune cell triggering and functional expression against coronavirus.
\end{abstract}

\section{Introduction}

The World Health Organization had announced (COVID-19) as a pandemic on 11 March 2020[1]. The epidemic evolves and spreads resulting in highly rates of infection or even death. While the vaccine is absent, there is an essential need to mention the immune system, nutrition and how could we enhance ourselves against viral deadly infections especially among vulnerable patients as persons aged more than 65 years, with worthily health conditions (asthma, heart, chronic disease, or even immunosuppressed). COVID19 may be transferred either by directly with infected patients or indirectly contact with surfaces used by the infected patients[2]. COVID-19 can cause a cytokine storm, which includes serious burst in the inflammatory ([IL]-6, IL-1 $\beta$, (TNF)- $\alpha$ ) (IFN)- $\gamma)[3]$. Undoubtedly, healthy diet, specific nutrients and nutrient facts can be effective with respect to disease vulnerability and immune responses by gene expression, cells activation, molecules production and gut microbial discourages [4].

Adjuration protection procedures under quarantine, self-isolation and sterilization are recommended for protection of COVID-19 infections.

Since being under quarantine, people seek for boredom and fear; due to the unemployment and news tragedy by social media platforms. This might result in poor diet quality and mood negative effect. Moreover, the unhealthy diet could increase inflammation, obesity, diabetes, cancer, lung problems and even the risks for more serious diseases than CoVID-19[5].

As a consequence, it is deemed crucial to consume serotonin and synthesis food sources such as almond, banana, oat and cherry to get rid of quarantine stresses[6]. In addition, tryptophan sources can act as sleep-inducing, neuropeptide Y inhibition, killer cell activity generator and respiratory infections control[7]. Furthermore, dairies, specially skimmed yogurt is strongly recommended for its highly useful microorganisms to ameloriate immunity system strengthens[8]. During quarantine, the nutritional deficiencies of macronutrients and micronutrients intake indeed resulted in depression of the immune function as secretory antibody, phagocyte, cytokine production eventually, increase the susceptibility for viral infections as COVID-19[9]. 
(Fig. 1) Shows the nutrients intake for novel Covid-19 Era effect on functions related and virus targeted under quarantine. Vegetables, salads and fruits regularly intake can supply micronutrients as vitamin (A, B, C, D, E), minerals ( $\mathrm{Zn}, \mathrm{Se}, \mathrm{Cu}, \mathrm{Fe}, \mathrm{Ca}$ ) and phytochemicals (rutin, hesperidin) to clean the body and boost the immune function[10].

Additionally, antioxidants food sources can increase (T-cell subsets), interleukin-2, lymphocyte reaction against the mitogen and even the activity of natural killer cell (NK cells)[11]. There are a lot of nutritional supplements to motivate the immune system due to its bioactive ingredients such as bee pollen, echinacea, mulone syrup, thyme and (nigella sativ, cod liver, pumpkin seed, fennel) oils[12]. It is necessary to reduce sugars intake as they reduce white blood cells function against microbes[13]. Admittedly, smoking is forbidden due to the free radical, nicotine and nitrogen dioxide ozone productions, which in turn weakens immunity[14].

The current article focuses on the immunenutrition relationship with the respiratory viruses as coronavirus. Definitely there are numerous nutritional date of enhancing the complex immune system needs, in synergy or individually such as diet, fruit, herbs, vitamins, nutraceuticals, antioxidants, trace elements and probiotics with the help of light sports against respiratory infections and pneumonia as COVID-19 syndromes.

\section{Essential Nutrients for Healthy Respiratory System}

\section{Vitamin A}

"Anti-infective" is a common name due to its enormous functions against measles-related pneumonia, human immunodeficiency virus (HIV), lung diseases and bronchitis virus[15]. Retinol plays a basic function in the immune system due to the angiotensin enzyme, which is essentially needed for SARS/COV-2 body entry[16].

\section{Vitamin B group}

Vitamin B group is famous for its individual function. Riboflavin is essential for cell energy metabolisms against (MERS-CoV)[17]. Niacin can kill Staphylococcus aureus and inhibit the neutrophil infiltration with a physically powerful anti-inflammatory activity in ventilator-induced lung injury[18]. Pyridoxine is well known for the immune system due to the protein metabolism function[19]. Folic acid can enhance the immune system by white blood cell production that acts as soldiers for body defiance. Cobalamin remains the ratio $\left(\mathrm{CD}^{4+/} \mathrm{CD}^{8+}\right)$ balances and facilitates (T-lymphocytes cells) production in a normal range[20]. Consequently, the Vitamin B group might be used as COVID-19 treatment.

\section{Vitamin $\mathbf{C}$}

Vitamin $\mathrm{C}$ is beneficial in pneumonia treatment, cellular functions, collagen synthesis in connective tissues, immune support against pathogens and oxidative stress[21]. Vitamin C is well-known as an antioxidant that can offset oxidative stress effects by reducing antioxidant enzymes and consequently oxidative damage increase[22]. Vitamin C may act as a weak antihistamine agent to aid COVID-19 syndromes such as running nose, swollen sinuses and sneezing, which is highly found in several fruits and vegetables like oranges, broccoli and blackcurrants.

\section{Vitamin D}

Vitamin D acts as a hormone, that may be synthesized by the sunlight through the body to enhance immune cells and maintain bone integrity[23]. Vitamin D is considered as a safe strategy to protect from respiratory viruses and increase the antiviral defenses. vitamin D low levels may modulate the expression as (chemokines, interferon, tumor necrosis and proinflammatory cytokines). COVID-19 infected patients almost might have an unsatisfactory vitamin D level. As a result, vitamin D might act as an additional beneficial choice for coronavirus treatment by getting $(10 \mu \mathrm{g})$ as a supplement daily[24].

\section{Vitamin E}

Vitamin E acts as an antioxidant against free radicals to reduce oxidative stresses. Vitamin E lower intake can cause of coxsackievirus B3 myocardial injury infection and bovine coronavirus in mice and calves, respec- 
tively[25]. In addition, vitamin E supplements induce effective thymic atrophy early recovery of especially [?] 60 years[26].

\section{Zinc}

Zinc is involved in preventing pneumonia, inflammatory responses, immune system (adaptive and innate cells) and pathogen-eliminating[27]. Moreover, the low concentrations intake of pyrithione and zinc can inhibit (SARS-CoV) and torquetenovirus (TTV)[28]. As a result, zinc supplements as UL of $25 \mathrm{mg} /$ day and naturally crab, shrimps, eggs and adzuki beans can control coronavirus (COVID-19) symptoms.

\section{Selenium}

Selenium is well known due to the functions of selenoproteins as an antioxidant defense for cell signaling as (CAT, SOD, GSH, ROS) and redox homeostasis[29]. Glutathione peroxidase is famous for reducing lipid peroxides and hydrogen, thioredoxin reductase for the thiol systems homeostasis. ROS are generated against (HIV) for the immunity system, (RSV) for the respiratory system and flu viruses 30]. Selenium low intake level can weaken the immune system, on the other hand rapid RNA mutations from benign variants to virulence. Ginseng bioactive component extract had used successfully for bronchitis coronavirus. As a result selenium supplementation can be used for COVID-19 treatment[31].

\section{Copper}

Copper is essential for the metalloenzyme control like superoxide dismutase, which has a vital function in the immunity system as ( $\mathrm{T}$ and $\mathrm{B}$ ) lymphocyte responses. Copper supplementation regular intake for eight weeks established suppressed mitogen-induced lymphoproliferative response[32].

\section{Iron}

Iron salts are required for enhancing immunity like iron lactoferrin and catalase. Respiratory infections, anemia and diarrheal are obvious syndromes for iron deficiency[33].

\section{Calcium}

Calcium works mainly on lymphocytes, which helps lymphocytes to maintain $\left(\mathrm{Ca}^{2}\right)$ as low levels. In addition, vitamin D and calcium cooperation can affect on several types of immune cells like antigen-presenting, $(\mathrm{T})$ and (B) cells to adjust both adaptive and innate immune responses, that may extend further than calcium and bone homeostasis.[34].

Therefore, vitamins and minerals as principal nutrients for immunonutrition might be useful for COVID-19 prevention.

\section{Declaration of Competing Interest}

There are no conflicts to declare

\section{FIGURE CAPTION:}

Fig. 1 Nutrients intake for novel Covid-19 Era effect on functions related and virus targeted under quarantine.

\section{References}

1- Zhu N, Zhang D, Wang W, Li X, Yang B, Song J, et al. A novel coronavirus from patients with pneumonia in China, 2019. N Engl $J$ Med .2020; 382: 727-33.

2- Ong SWX, Tan YK, Chia PY, Lee TH, Ng OT, Wong MSY, et al. Air, surface environmental, and personal protective equipment contamination by severe acute respiratory syndrome coronavirus 2 (SARS-CoV-2) from a symptomatic patient. JAMA. 2020.

3- Wang W, He J, Lie p, Huang l, Wu S, lin Y, et al. The Definition and Risks of Cytokine Release SyndromeLike in 11 COVID-19-Infected Pneumonia Critically ill Patients: Disease Characteristics and Retrospective Analysis. Med Rxiv. 2020. 
4- Valdés-Ramos R, Martínez-Carrillo BE, Aranda-González II, Guadarrama AL, Pardo-Morales RV, Tlatempa P, et al. Diet, exercise and gut mucosal immunity. Proc Nutr Soc. 2010; 69: 644-50.

5- Wu C, Chen X, Cai Y, Xia J, Zhou X, Xu S, et al. Risk factors associated with acute respiratory distress syndrome and death in patients with coronavirus disease 2019 pneumonia in Wuhan, China.JAMA Intern Med. 2020.

6- Peuhkuri K, Sihvola N, Korpela R. Diet promotes sleep duration and quality. Nutr Res. 2012; 32: 309-319.

7- Makino S, Ikegami S, Kume A, Horiuchi H, Sasaki H, Orii N. Reducing the risk of infection in the elderly by dietary intake of yoghurt fermented with Lactobacillus delbrueckii ssp. bulgaricus OLL1073R-1. $\mathrm{Br} J$ Nutr. 2010; 104: 998-1006.

8- Conlon M, Bird A. The impact of diet and lifestyle on gut microbiota and human health. Nutrients. 2015; $7(1): 17-44$.

9- Gleeson M, Nieman DC, Pedersen BK. Exercise, nutrition and immune function. J Sports Sci 2004; 22: $115-125$.

10- Dhandevi P, Jeewon R. Fruit and vegetable intake: benefits and progress of nutrition education interventions-narrative. Iranian journal of public health. 2015; 44(10): 1309-1321.

11- Sami R, Chun-Juan L, Yan Z, Ying L, Chang-Hao S. Phytochemicals, Antioxidant and Anti-inflammatory Potency Activities of Cabbage(Brassica oleracea L. var. capitata) . Asian Pacific Journal of Cancer Prevention. 2014; 14(11): 6657-6662

12- Haider IA, Sarmad GA, Hassan NH. The Effect of Nutrition on Immune System Review Paper. Food Science and Quality Management. 2019; 90: 31-53

13- Munkyong P, Simin M, Dayong Wu. The Role of Nutrition in Enhancing Immunity in Aging. Aging and Disease. 2012; 3 (1): 91-129.

14- Krause D, Mastro AM, Handte G, Smiciklas-Wright H, Miles MP, Ahluwalia N. Immune function did not decline with aging in apparently healthy well-nourished women. Mechanisms of ageing and development. 1999; 112(1): 43-57.

15- Jee J, Hoet AE, Azevedo MP, et al. Effects of dietary vitamin A content on antibody responses of feedlot calves inoculated intramuscularly with an inactivated bovine coronavirus vaccine. Am J Vet Res. 2013;74: $1353-1362$.

16- Sinha S, Cheng K, Aldape K, Schif E, Ruppin E. Systematic Cell Line-Based Identification of Drugs Modifying ACE2 Expression.Preprints. 2020.

17- Keil SD, Bowen R, Marschner S. Inactivation of Middle East respiratory syndrome coronavirus (MERS-CoV) in plasma products using a riboflavin-based and ultraviolet light-based photochemical treatment.Transfusion. 2016; 56: 2948-2952.

18- Jones HD, Yoo J, Crother TR, et al. Nicotinamide exacerbates hypoxemia in ventilator-induced lung injury independent of neutrophil infiltration. PLOS One. 2015; 10: e0123460.

19- Kyme P, Thoennissen NH, Tseng CW, et al. C/EBPepsilon mediates nicotinamide-enhanced clearance of Staphylococcus aureus in mice.J Clin Invest. 2012; 122: 3316-3329.

20- Kathleen M and Vasso A. Vitamin B12, Folic Acid, and the Immune System.Nutrition and Immunity. $2019 ; 103-114$.

21- Carr A, Maggini S. Vitamin C and immune function.Nutrients. 2017; 9: 1211.

22- Hosakote YM, Jantzi PD, Esham DL, et al. Viral-mediated inhibition of antioxidant enzymes contributes to the pathogenesis of severe respiratory syncytial virus bronchiolitis. Am J Respir Crit Care Med. 2011; 
183: $1550-60$.

23- Martineau AR, Jolliffe DA, Hooper RL, Greenberg L, Aloia JF, Bergman P, Dubnov-Raz G, Esposito S, Ganmaa D, Ginde AA et al. Vitamin D supplementation to prevent acute respiratory tract infections: systematic review and meta-analysis of individual participant data.BMJ. 2017; 356: i6583.

24- Lei Z and Yunhui L. Potential interventions for novel coronavirus in China: A systematic review. J Med Virol. 2020; 92: 479-490.

25- Nonnecke BJ, McGill JL, Ridpath JF, Sacco RE, Lippolis JD, Reinhardt TA. Acute phase response elicited by experimental bovine diarrhea virus (BVDV) infection is associated with decreased vitamin D and E status of vitamin-replete preruminant calves. J Dairy Sci. 2014; 97: 5566-5579.

26- Moriguchi S and Muraga M. Vitamin E and immunity. Vitam Horm. 2000; 59: 305-36.

27- Gammoh NZ, Rink L. Zinc in infection and inflammation.Nutrients. 2017; 9: E624.

28- Iovino, L., et al., High-dose zinc oral supplementation after stem cell transplantation causes an increase of TRECs and CD4+ naive lymphocytes and prevents TTV reactivation. Leuk Res. 2018; 70: 20-24.

29- Labunskyy, V.M.; Hatfield, D.L.; Gladyshev, V.N. Selenoproteins: Molecular pathways and physiological roles. Physiol. Rev. 2014; 94: 739-777.

30- Molteni, C.G.; Principi, N.; Esposito, S. Reactive oxygen and nitrogen species during viral infections. Free Radic Res. 2014; 48: 1163-1169.

31- Ma X, Bi S, Wang Y, Chi X, Hu S. Combined adjuvant effect of ginseng stem-leaf saponins and selenium on immune responses to a live bivalent vaccine of Newcastle disease virus and infectious bronchitis virus in chickens. Poult Sci. 2019; 98: 3548-3556.

32-Chakraborty P, Das S, Banerjee K, Sinha A, Roy S, Chatterjee M, Choudhuri SK. A copper chelate selectively triggers apoptosis in myeloidderived suppressor cells in a drug-resistant tumor model and enhances antitumor immune response. Immunopharmacology and Immunotoxicology. 2014; 36(2): 165-175.

33- Chandra RK. Impact of nutritional status and nutrient supplements on immune responses and incidence of infection in older individuals. Aging Research Reviews. 2004; 3(1): 91-104.

34- Monika V and Jean-Pierre K. Calcium signaling in immune cells. Nat Immunol. 2009; 10(1): 21-27. 


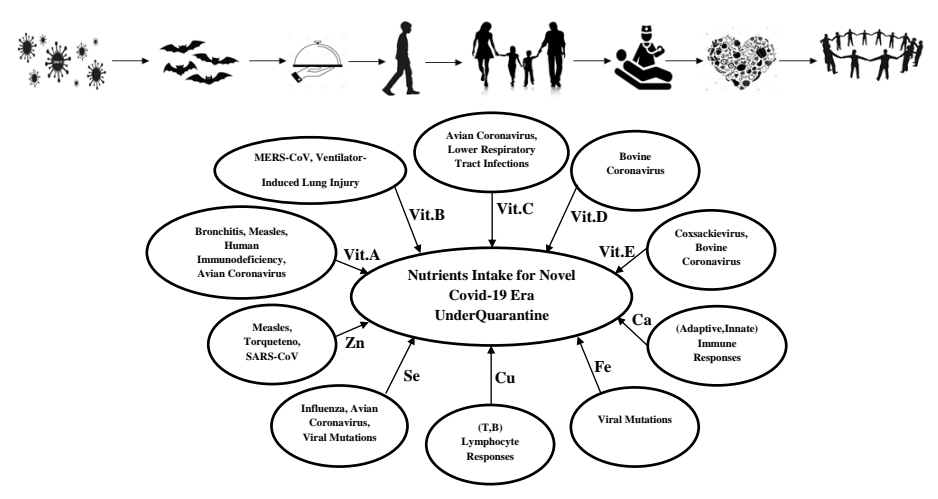

